\title{
Summary of Revisions for the 2010 Clinical Practice Recommendations
}

B eginning with the 2005 supplement, the Clinical Practice Recommendations contained only the "Standards of Medical Care in Diabetes" and selected other position statements. This change was made to emphasize the importance of the "Standards" as the best source to determine American Diabetes Association recommendations. The position statements in the supplement are updated yearly. Position statements not included in the supplement will be updated as necessary and republished when updated. A list of the position statements not included in this supplement appears on p. S100.

\section{Additions to the "Standards of Medical Care in Diabetes"}

- A section on cystic fibrosis-related diabetes has been added.

\section{Revisions to the "Standards of Medical Care in Diabetes"}

In addition to many small changes related to new evidence since the previous version, the following sections have undergone major changes:

- The section "Diagnosis of diabetes" has been revised to include the use of AlC to diagnose diabetes, with a cut point of $\geq 6.5 \%$.

- The section previously titled "Diagnosis of pre-diabetes" has been renamed "Categories of increased risk for diabetes." In addition to impaired fasting glucose and impaired glucose tolerance, an AlC range of $5.7-6.4 \%$ has been included as a category of increased risk for future diabetes.

- The section "Detection and diagnosis of GDM" has been revised to discuss potential future changes in the diagnosis based on international consensus. $\bullet \bullet \bullet \bullet \bullet \bullet \bullet \bullet \bullet \bullet \bullet ~$
DOI: $10.2337 / \mathrm{dc} 10-\mathrm{S} 003$

(C) 2010 by the American Diabetes Association. Readers may use this article as long as the work is properly cited, the use is educational and not for profit, and the work is not altered. See http://creativecommons. org/licenses/by-nc-nd/3.0/ for details.
- The section "Diabetes self-management education" has been extensively revised to reflect new evidence.

- The section "Antiplatelet agents" has been extensively revised to reflect recent trials questioning the benefit of aspirin for primary cardiovascular disease prevention in moderate- or low-risk patients. The recommendation has changed to consider aspirin therapy as a primary prevention strategy in those with diabetes at increased cardiovascular risk (10-year risk $>10 \%)$. This includes men $>50$ years of age or women $>60$ years of age with at least one additional major risk factor.

- The section "Retinopathy screening and treatment" has been updated to include a recommendation on use of fundus photography as a screening strategy.

- The section "Diabetes care in the hospital" has been extensively revised to reflect new evidence calling into question very tight glycemic control goals in critically ill patients.

- The section "Strategies for improving diabetes care" has been extensively revised to reflect newer evidence. 